\title{
Reales Marketing führt die Kunden zum Kauf
}

Wir brauchen reales Marketing, das sich auf reales Kundenverhalten stützt. Interne Marketing- und Vertriebsprozesse im Unternehmen sind erst effektiv und effizient, wenn sie sich an den Kundenprozessen und -transaktionen ausrichten. Fein ausgedachtes Imagemarketing in der Gedankenwelt des Kunden wird überschätzt und führt zur Verschwendung. Gut positionierte und attraktive Markenauftritte bewegen den Kunden nämlich selten zur Kaufhandlung.

Christian Belz, Marc Rutschmann 

Dieser Beitrag erfasst Ansatz, Regeln, Handwerk und Manigement des realen Marketing. Empirisch stützt er sicl auf rund 200 Verhaltensanalysen von Käufern in den verschiedensten Wirtschaftszweigen und praktische Anwendungen.

\section{Welten der Identifikation und der Handlung im Marketing}

Seit vielen Jahren betonen Experten im Marketing die gleiche Ausgangslage. Die Produkte sind auswechselbar, der Preisdruck steigt und die Vielfalt an Informationen und Angeboten ist überbordend. Zwei grundsätzliche Antworten lassen sich in der Praxis beobachten.

Abbildung 1 zeigt zwei Inserate, die diese Antworten andeuten. In beiden Inseraten handelt es sich um Waschmaschinen von Schulthess. Links in der schönen Welt des Marketing mit dem Model Fiona Hefti (eine frühere und bekannte Miss Schweiz). Rechts in der Handlungswelt des Kunden mit dem Handelsinserat des führenden Händlers für Heimelektronik Dipl. Ing. Fust AG, dort auch mit der Figenmarke „Fust by Schulthess“.

Beide angedeuteten Welten lassen sich professionell verfolgen. In kurzer Form ist die Umschreibung:

1. Identifikationswelt des Marketing: Für Empfänger wirkt das Marketing in der Identifikationswelt ästhetisch, attraktiv, kreativ, manchmal humorvoll und indirekt oder übergeordnet. Unternehmen und ihre Angebote sollen aus dem Meer von Produkten und lnformationen positiv herausragen. Die ausgelösten guten Gedanken und Gefühle des Kunden sollen ihn schließlich zum Kauf bewegen. Schlüsselworte in der Diskussion sind Positionierung, Bekanntheit, Image und Marke, Inszenierungen und Emotionalisierung. Anbieter bestimmen eine klare Kommunikations-

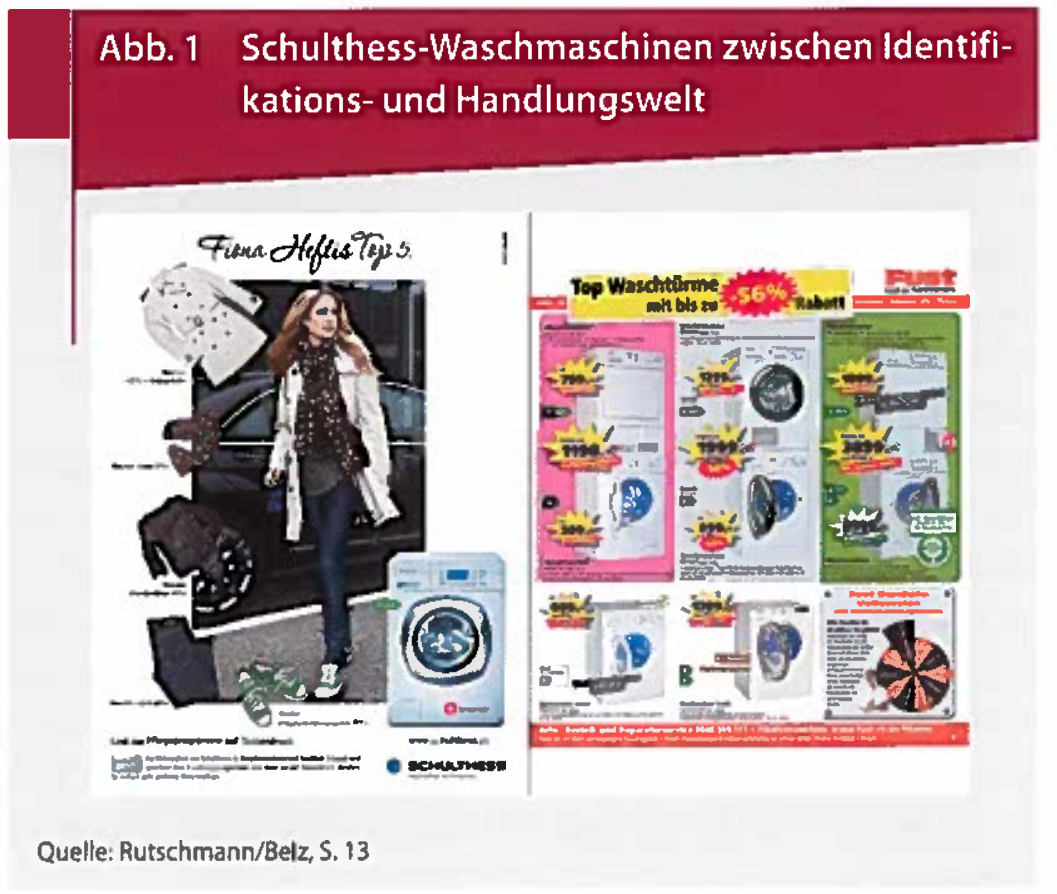

Prof. Dr. Cliristian Belz

ist Ordinarius für Marketing an der Universitat St. Gallen und Direktor am Institut für Marketing.

\section{Dr. Marc Rutschmant}

ist Whaber der Dr. Marc Rutschmam AG Ziirich und Lehrbeauftragter an der Universitäl st. Gallen.

\author{
Christian Belz \\ Universităt St. Gallen, St. Gallen, Schweiz \\ E-Mail: christ an belz@unisg.ch
}

Marc Rutschmann

Dr. Marc Rutschmann AG, Zürich, Schweiz

E-Mall: marc.rutschmannemrutschmann.com 
strategie und sichern top-down sehr rigoros ihre Corporate Identity. Instrumente sind hau ing Inserate-Kampagnen, TV-Spots oder Firmenbroschüren. Hankierend suchen witzige Youtube-Filme möglichst viele Betrachter, um damit irgendwie auf den Markenwert einzuzahlen. Die Identifikation des Kunden ist in dieser Argumentation die Eintrittskarte für spätere Kuufprozesse.

2. Handlungswelt des Marketing: Maßnahmen in der Handlungswelt des Marketing wirken eher plump, simpel, manipulativ, manchmal spielerisch und vor allem direkt. Es gilt, überlastete Kunden mit vielen Kaufabsichten und meist langen und verschlungenen Wegen in Schritten zum Kauf zu fuhren. Schlüsselwörter in dieser Welt des Marketing sind Kundenprozesse, Kaufmuster, Response, Konversion und Handlungsauslösung. Der Ansatz ist stark bottom-up geprägt und stützt sich auf konkrete Kaufprozesse und -laandlungen der Kunden. Marketing in der Handlungswelt ist eher in der Werbung des Einzelhandels, im Direktmarketing, im Call-Center oder im Vertrieb anzutreffen. Einziger Bezug ist die Wirksamkeit; Umsatzund Ertragsziele stehen im Vordergrund. Kunden handeln bei gleichwertigen Angeboten auch ohne Identifikation und lassen sich durch situative Gelegenheiten und den puren Reiz der Handlung beeinflussen.

Naturgemaß kombinieren Unternehmen diese Welten. Im positiven Fall verstärken sje sich gegenseitig. Wir sind aber überzeugt, dass Marketing in der Handlungswelt gewinnt und die Identifikationswelt in der aktuellen Diskussion weit überschätzt wird (Belz/Rutschmann 2014, S. 2 ff.). Besonders in Märkten, bei denen Kunden kaum mehr Kaufrisiken einge hen und parallel sehr viele Optionen gedanklich verfolgen, wird die Handlung des Kunden zum Engpass. Zwar gibt es viele starke Marken, sie werden aber eher durch Kundenhandlungen aufgebaut, als dass große Markenkampagnen die Kundenhandlungen bewirken. Erfolgreiche Marken wie Google, Amazon oder Apple lassen sich als Beispiele dafur anfuhren.

Natürlich trifft unsere Argumentation auf besondere Märkte weniger zu. Beispielsweise spielt bei sozialen Produkten (etwa Autos, Kleidung oder Uhren) die Identifikation eine stärkere Rolle. Auch scheinen (europäische) Marken in asiatischen Ländern besonders wichtig zu sein. Obschon die Verhaltensanalysen zu Kundenprozessen bisher mehrheitlich für Konsum-, Gebrauchsgüter und Dienstleistungen durchgeführt wurden, triftt die Argumentation noch stärker für Industriemärkte zu. Diese Prozesse sind noch weit komplexer und schließen verschiedene Personen des Kunden ein. Den Ansatz konsequent in die Industrie zu bringen, bleibt eine nächste, große Aufgabe.

\section{Reales Marketing}

Reales Marketing nennen wir die neuen Ansätze in der Handlungswelt des Kunden. Diese Ansätze selektieren Kunden mit hoher Erfolgswahrscheinlichkeit und konzentrieren sich auf wenige Hebel im Prozess, um Kunden zum Kauf zu führen. Reales Marketing beruht auf einem konsequenten Bottomup-Vorgehen und nutzt bisherige Verhaltensmuster und -mechanismen. Der Gegenpol ist das klassische Identifikationsmarketing, alten voran die Markenfuhrung. Gegenpool ist auch eine Marktforschung, die lediglich die Interpretationen des Kunden erfasst, warum er kauft oder nicht kauft.

\section{„Unternehmen und ihre Angebote sollen aus dem Meer von Produkten und Informationen positiv herausragen."}

\section{Zwei Stränge prägen das reale Marketing:}

1. Customer Relationship Management (CRM): Mit analytischem Customer Relationship Management erkennen Unternehmen aus den Transaktionen ihrer Kunden die prägenden Kaufmuster. Gestützt auf Datamining lassen sich die wichtigen Kaufanlässe oder Brüche für Kunden bestimmen und besonders Kunden selektionieren, die für spezifische Angebote empfanglich sind. Der "Closed Loop" des Database-Marketing besagt dabei, dass jede Kundenreaktion (beispielsweise auf Aktionen), diese Database wieder verbessert. Die Hinweise zu Big Data zeigen, dass sich die eigenen Informationen über Kunden inzwischen wertvoll anreichern und auswerten lassen. Marketingmaßnahmen und ihr Erfolg lassen sich im CRM und Direktmarketing messen und damit steuern.

2. Kaufprozessorientiertes Marketing: Mit Micro-Verhaltensanalysen der Kunden werden die Schritte des Kunden zum Kauf detailliert aufgezeichnet. Es wird dabei ersichtlich, wo viele Kunden ihren Prozess weiterführen, verzögern oder abbrechen. In diesen Prozessen lassen sich die Stellen oder Hebel erkennen, bei welchen Marketing erfolgreich ansetzen kann, um den Kunden weiter zum Kauf zu führen. Auch fär Unternehmen, die Lösungen statt Produkte für Kunden 
anbielen wollen, spielt der Weg zum Kauf für unterstützende Services eine entscheidende Rolle.

Diese Ansātze lassen sich getrennt verfolgen, sind aber auch ein wirksames Gespann. CRM liefert eher die Hinweise zur Kundenselektion und -gruppierung und berücksichtigt auch besonders wertvolle Kunden für das Unternehmen. Kaufprozesse bezeichnen die konkreten Schritte des Kunden, auch solche, die über Interaktionen mit den Unternelımen hinausgehen, und sie weisen den Weg für wirksame Inhalte in Marke tingmaßnahmen. Diese Prioritäten werden auch im Bericht des Marketing Science Institutes (2014) verankert, die an erste Stelle „Understanding Customers and the Customer Expe rience" sowie an die zweite Stelle "Developing Marketing Analytics for a Data-Rich Environment" für die zukünftige Entwicklung in Forschung und Praxis des Marketing setzen.

Flankiert werden die erwähnten Ansätze durch ganzheitliche Erlebnisse des Kunden. Dabei denken wir an Events, Messen und Fabrikbesuche. Eine Sonderstellung nimmt die eigene Initiative des Kunden ein, welche sich durch Inbound Marketing nutzen lässt. Es fördert und nutzt die Initiative des Kunden. Weil der Kunde selbst handelt und sich an das Unternehmen wendet, ist es „Volltreffermarketing“.

Schließlich spielen abgegrenzte Experimente und Tests eine wichtige Rolle im realen Marketing. Oft zeigen nur sie, was wirklich funktioniert. Dabei denken wir nicht nur an geprüfte Varianten von Direct Mails. Umfassendere Lösungen lassen sich auch in einzelnen Ländern oder Regionen, ausgewählten Produktbereichen oder in spezifischen Filialen erproben.

\section{Spielregeln des realen Marketing}

Gibt es grundlegende Spielregeln, die das reale Marketing im Vergleich zu klassischem Marketing prägen? Folgende zehn Regeln setzen die neuen Gewichte. Sie krempeln manche herkömmlichen Argumente im Marketing um (Rutschmann/ Belz 2014, S. 23 f.):

1. Kundenhandlung vor Identifikation: Die Kundenhandlung ist wichtiger und qualifizierter als eine ldentifikation des Kunden.

2. Schlüsseltreiber vor gesuchter Einzigartigkeit: Im Prozess sind gut genutzte Treiber im Kernnutzen der Kunden bedeutender als gesuchte Unique Selling Propositions.

3. Kundenprozess vor Instrumenten und Medien: Der Kundenprozess mit seinen Hebeln steht vor dem Einsatz der vielfâltigen Instrumente der Markıbearbeitung (von Inse raten bis Social Media); daraus ergibt sich ein wirksames Zusammenspiel, weil jedem Instrument die Aufgabe im Kundenprozess zugewiesen ist.

4. Sclıritte vor dem Kauf vor einer Fixierung auf den Kaufakt: Reales Marketing konzentriert sich auf die vielen Schritte des Kunden vor dem Kauf mit ihren differenzierten Anstôßen, Befindlichkeiten und Bedürfnissen. Es ist nicht mehr auf den Kaufakt fixiert, bei welchem Kunden vermeintlich Vor- und Nachteile des Angebotes kritisch abwägen.

5. Dialog vor Monolog und Inbound vor Outbound: Kommunikation ist dialogisch und fördert die Zustimmungen und Zwischenhandlungen des Kunden. Initiativen des Kunden werden im lnbound Marketing aktiv gefördert und genutat.

6. Bottom-up vor top-down: Kundenprozesse werden konsequent bottom-up erfasst, um Hebel zu erfassen und Marketing sowie Vertrieb wirksam einzusetzen. Nur fein ausgedachte Top-down-Konzepte verlieren an Bedeutung.

7. Moderation vor Invasion: Marketing moderiert die Kundenprozesse in vielfältigen Bedingungen. Es nutzt und schafft situative Gelegenheiten. Märkte auszurollen oder gewagte Strategien umzusetzen bleibt meistens illusionär; mindestens, wenn nicht kraftvolle Innovationen vermarktet werden können.

8. Differenzierung vor Integration: Wichtigste Herausforderung des Marketing ist es, nahe beim Kunden vorzugehen und vielfältige Konstellationen von Leistungen und Kunden zu bewältigen. Im Vergleich hilft eine abstrakte Integration kaum.

9. Zusammenarbeit am Kundenprozess vor Spezialisierung: Vielfältige Spezialeinheiten und externe Dienstleister im

\section{Zusammenfassung}

Kunden gefällt vieles und sie haben zahlreiche Absich ten. Nur werden ihre Wege zum Kauf immer länger, sie werden oft abgebrochen, verschoben oder verändert. Es ist deshalb die Kernaufgabe für Marketing und Vertrieb, sehr konkret die realen Kaufprozesse zu erfassen und die Hebel zu erkennen, an denen sich Kunden erfolgreich zum Kaufabschluss und zum Wiederkauf führen lassen. 
Marketing mússen eng zusanmenarbeiten, statt gegeneinander zu kämpfen oder nebeneinander zu wirken. Kaufprozesse sind (neben den Unternehmensstrategien) die wirksame Ausrichtung für alle Teildisziplinen und danit beispielsweise der /ugang fur Lösungen im Bereich Crossmedia und Multichinnel.

10. Dezentrale Marketingarbeit vor zentralem Spaß: Wirksame Marketingarbeit ist dezentral, kundennah, konkret. Dieses Marketing macht mehr Arbeit und lässt sich schtecht an Dienstleister delegieren. Dagegen ist es eher ein Spaß, zentrale Kampagnen zu verabschieden und ausrollen zu lassen.

Und: Umsat 2 und Frtrag sind die Ziele des Marketing und nicht irgendwelche Wirkungen im Kopf des Kunden. Einstellungen oder Motive sind zu wenig verhaltenswirksam. Zwischenziele müssen sich auf spexifische Hebel im Kundenprozess konzentrieren.

In der Folge konzentrieren wir uns auf den genannten Strang des Kaufprozessorientierten Marketing. Zu CRM findet sich bereits eine verbreitete Basis.

\section{Anspruchsvolles Handwerk}

Mit einem geeigneten Vorgehen lassen sich Zwischenschritte im Kaufprozess um den Faktor 3 bis 8 für Anbieter verbessern, wie zahlieiche Anwendungen belegten. Offensichtlich lässt sich der häufig beklagte Wirkungsverlust vieler Marke- tingnaßnahmen auffangen (Rutschmann 2005, S. 61 ff.). Nur genügen Ansatz und Argumentation nicht. Kundenprozessorientiertes Marketing stutat sich auf ein anspruchsvolles Handwerk.

Zuerst gilt es, reale Kundenprozesse zu erheben. Befragte Kunden beschreiben detailliert, welche Schritte sic an welchen Orten durchliefen, um ein Produkt zu kaufen oder auf einen Wettbewerber umzuschwenken. Solche Prozessbeschreibungen der Kunden dauern oft eine oder zwei Stunden. Sie werden aufgezeichnet und codiert. Viete Prozesse werden ubereinandergelegt, qualitativ und quantitativ ausgewertet, nach Zietgruppen differenziert. Bereits für Konsum- und Gebrauchsgüter brauchen Kunden 40 bis 50 Schritte. Daraus lassen sich die prägender Muster für einen Kauf und Nichtkauf erkennen und vergleichen sowie zwei bis fünf Hebel identifizieren, an denen Marketing und Vertrieb ansetzen sollen, um Kundenprozesse zu erleichtern und wirksam fortzusetzen.

Abbildung 2 zeigt als Beispiel die Kaufprozessanalyse für $\mathrm{Ha}$ milien und junge Paare, die ihre Ferien bei Hotelplan buchten. Die vielen Schritte sind nicht erkenntbar, deuten aber an, wie fein die Schritte erfasst wurden.

Vorteil dieser Verhaltensanalysen ist es, dass alle wesentlichen Hinweise für die Schritte des Kunden aktuell erfasst werden. Sollten beispielsweise Marken oder Recherchen im Internet in einem Schritt wichtig sein, so erscheinen sie. Gleichzeitig sind die Ergebnisse solcher Analysen nach unserer Erfahrung sehr robust.

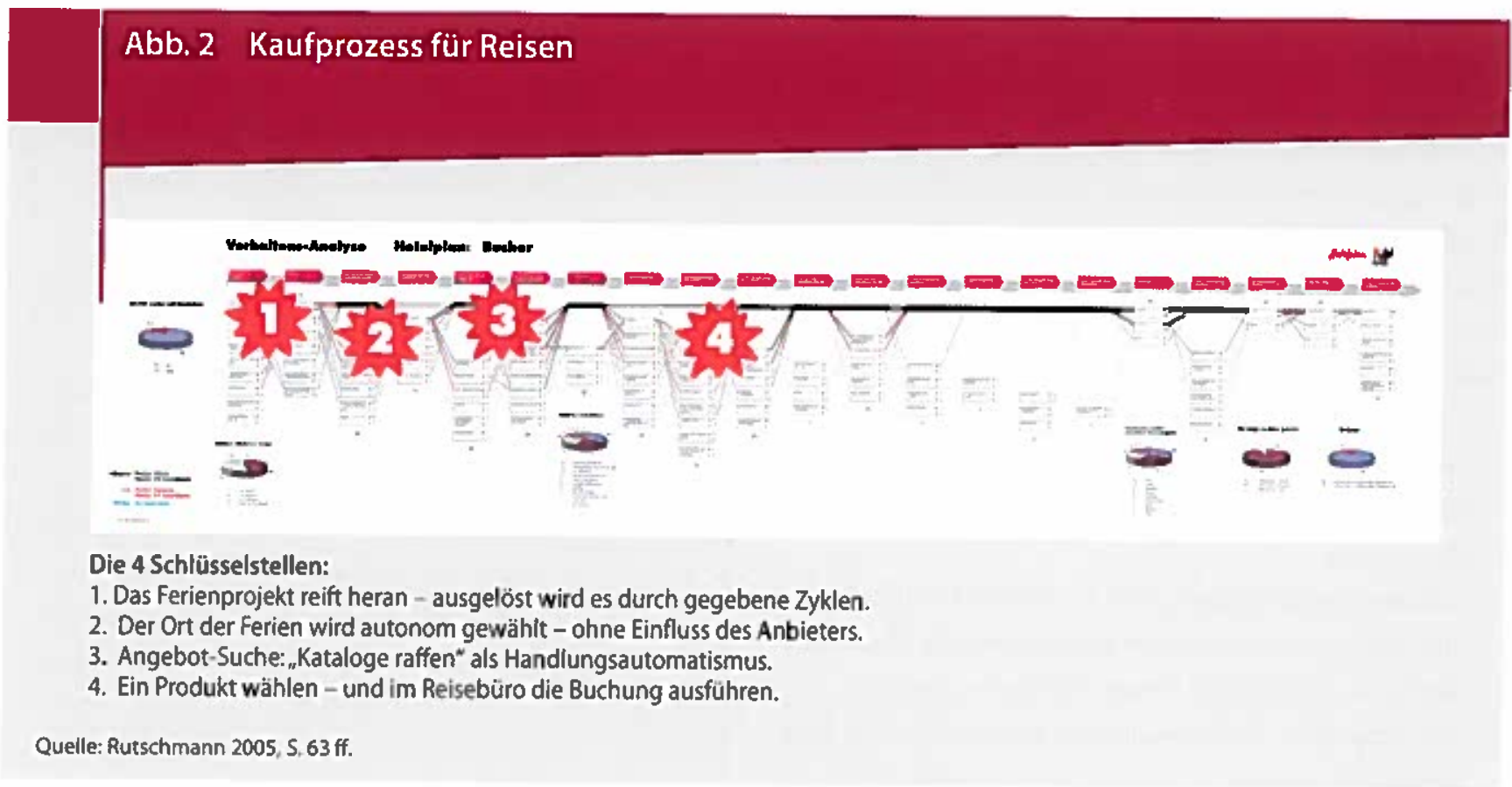


Diese Analyse zeigt, dass zu Beginn des Prozesses die Destination in Vordergrund stcht und alle anderen Erwägungen überragt: die (bildlhafte) Vorstellung, wo und wie die schöne Urlaubszeit verbracht werden wird. Getrieben durch die Destination sucht der potenzielle Kunde entsprechende Kataloge. Vornehmliclı möchte er sie in Händen halten, in PrintForm, um sie mit seinen Liebsten durchblättern zu können. Wer ihm diese Ferien ermöglicht - Tui, Kuoni oder ein ande-

\section{„Zwar gibt es viele starke Marken, sie werden aber eher durch Kunden- handlungen aufgebaut, als dass große Markenkampagnen die Kundenhandlungen bewirken."}

rer - spielt in dieser Prozessphase keine Rolle. Kataloge müssen her; die Leistungsversprechen der Anbieter greifen ins Leere, etwa Garantieleistungen - die Erdbeben-Geld-zurückGarant Je beispielsweise oder Lifestyle-Attribute. Sicherheitsüberlegungen kommen allenfalls kurz vor dem Kaufabschluss ins Spiel. Aber welchen Verlauf der Prozess nimmt, zu welchem Anbieter er schließlich führt - diese Weichenstellung hat im Vorfeld stattgefunden, in der fruhesten Phase des Kaufprozesses. Dort gab der Treiber "Wo erhalte ich die Kataloge in Händen?" den Ausschlag.

Inzwischen führte die Dr. Marc Rutschmann AG (Zürich/ Schweiz) rund 200 Verhaltensanalysen durch, wichtig sind ebenso Beobachtungen der Prozesse. Leider lässt sich aber trotz dieser zahlreichen Analysen kein allgemeingültiger Kaufprozess herauslesen oder ein Baukasten der Kundenmuster entwickeln. Die Ergebnisse sind erst durch ihre Genauigkeit und Spezifität ergiebig. So unterscheiden sich beispielsweise die Kaufprozesse fur verschjedene Automarken von Toyota, Opel, Lexus bis BMW stark; sie sind spezifisch.

Die Analyse der Kaufprozesse zeigt, wo sich die Hebel befinden, um den Weg des Kunden zum Kauf fortzusetzen. An diesen Stellen gilt es nun, die konkreten Handlungen des Kunden auszulösen. Hier setzen die folgenden Wirkgesetze an.

\section{Wirkgesetze für erfolgreiche Kommunikation}

Sechs Wirkgesetze gilt es, fur eine wirksame Kommunikation zu beherzigen, die den Kunden erfolgreich zum Kauf führt (Rutschmann 2013, S. 59 ff.):
1. Angeborene Auslösemechanismen nutzen - Formen, Farben und Schemenbilder: Auf Kindergesichter reagieren wir automatisch. Gesichter sind ein Blickfang und erzeugen ganz bestimmte Emotionen; auch runde Formen erzeugen Aufmerksamkeit, ebenso wie schräge Balken ein Blickfang sind. Und wir reagieren stark auf Rot und auf Gelb. Es gibt manche angeborenen Mechanismen, die sich nutzen lassen.

2. Primat der Handlung - sofort belolnnen: Unwichtig, aus welchem Antrieb die Handlung des Kunden erfolgt Hauptsache, er handelt: Erst die Konsequenzen daraus prägen und determinjeren den weiteren Prozessverlauf. Rei\% der Handlung heißt die kurze Formel.

3. In wohlbemessenen Handlungsschritten ans Ziel: Das bedeutet, den Kunden nicht zu überfordern; ihn nur zu einer Handlung bewegen, zu der er ohnehin eine hohe Bereitschaft an den Tag legt, und ihn vielleicht nur einen kleínen Schritt dem finalen Ziel entgegenführen, der Kaufhandlung.

4. Commitments einholen - vom kleinen Ja zum großen Ja: Wichtig ist, immer wieder den Dialog anzustoßen: Der Kunde soll sich aussprechen; so führen wir ihn im Dialog in Richtung Zustimmungen (Commitments), die er uns gibt, zu immer mehr Zustimmungen - von der kleinen Zustimmung zur großen Zustimmung - bis ihm die Kaufhandlung als "logische" Konsequenz erscheint.

5. Dankesschuld aufbauen: Auf noch so kleine Vorleistungen reagieren wir mit einer empfundenen Dankesschuld - unterliegen gewissermaßen einem Zwang, etwas zurüickzugeben. Grundsätzlich gilt: Kleine Gaben vorab haben eine

\section{Kernthesen}

- Identifikationsmarketing geht von Mechanismer der Wirkung aus, die nur ausnahmsweise zutreffen.

- Reales Kundenverhalten lässt sich mit Micro-Verhaltensanalysen zum Kundenprozess und analytischem Customer Relationship Management Marketing erfassen und zeigt, wo Marketingmaßnahmen wirksam sind. - Der Erfolg von realem Marketing lässt sich an Beispielen belegen. Es braucht aber einen umfassenden Change-Prozess des Marketing, um die Vorteile für Unternehmen zu nutzen. 
höhere Wirkung als größere Gaben nach Vollzug der gewünschten Handlung.

6. Knappheit erzeugen: Das Angebot knapp zu halten, steigert die Handlungsbereitschaft. Beispiele: beschrankte Anzahl verfügbarer Produkte; güttig nur bis zu einem (knapp gehaltenen) Datum; deutlich machen, dass viele andere Mitmenschen dieses Angebot begehren: „Fin wohlhabender Ausländer habe ein Auge auf dieses Haus geworfen, sagt uns der Liegenschaftsmakler."

7. Sendervorteile nutzen: Gut erforscht sind die Merkmale, die den Beeinflussungserfolg eines Senders erhöhen. Das Wichtigste in Kürze: Einen lebendigen Sprecher, aus Fleisch und Blut, einsetzen. Die empfundene Ähnlichkeit. die Vertrautheit mit diesem Sprecher und das gute Aussehen können den Beeinflussungserfolg erhöhen. Die fachliche Autorität erhöht die Glaubwürdigkeit und seine Neutralität ist forderlich.

Gestützt werden diese Wirkgesetze auch durch eine gesicherte Verhaltensforschung und die Neurobiologie (zum Beispiel Cialdini 2010; Kahnemann 2012). Um diese Wirkprinzipien erfolgreich anzuwenden, braucht es eine neue Professionalität und gerichtete Kreativität.

In unserem Beispiel zu Hotelplan (Abbildung 2) führten die Erkenntnisse zu den Schlüsselstellen und den Wirkprinzipien zu einem neuen Marketing. Erstens wurde erkannt, dass Ferienprojekte bei Kunden autonom heranwachsen und nicht teuer beworben werden müssen. Zweitens wurde eine breite Katalogsuche in den Filialen (auch mit Wettbewerbsprodukten) massiv erleichtert. Das veränderte den Filialauftritt und die Besucherführung in einem Pilotbetrieb in Basel. Als neutrales Reisezentrum war die Filiale in Bezug auf Frequenz und Umsatz besonders erfolgreich. Zwar suchen Familien, Partner und ihre Freunde wieder selbst ihre Lösung aus den mitgebrachten Katalogen zu Hause, sie kehren aber zur Buchung in das entsprechende Geschäft zurück (Rutschmann 2005,

\section{Video}

Die Grundidee des Ansatzes findet sich auch in einem kleinen Film der Universität St. Gallen von rund $7 \mathrm{Mi}$ nuten: YouTube - Reales Marketing: Verkaufen statt Schaulaufen (oder englisch: Real Marketing: Stop Advertising and Start Selling).
S. 68 ff.). Schließlich helfen die Grundlagen des Direktmarketing dabei, dass Gestaltung, Sprache oder Wettbewerbe dass erwünschte Verhalten der Kunden fördern. Methoden des Fye-Trackings sind nützlich, um optimieren zu können, werden aber nur vereinzelt eingesetzt. Leider scheinen die Me-

\section{„Mit analytischem Customer Relationship Management erkennen Unternehmen aus den Transaktionen ihrer Kunden die prägenden Kaufmuster."}

chanismen zu professionellem Direktmarketing zunehmend verlernt 7.u werden, obschon sie ebenso im Internet-Marketing wichtig wären.

Nun lässt sich ein neuer Ansatz der Marktbearbeitung nicht einfach mit einzelnen, effolgreichen Maßnahmen und Aktionen begründen. Zudem treffen auch erfolgreiche Pilotprojekte vor einer Multiplikation auf mannigfaltige Widerstände. Es braucht einen grundsätzlichen Wandel.

\section{Change Management}

Einzelne Anwendungen in Unternehmen belegen eindrücklich, dass reales Marketing erfolgreich wirkt. Nur braucht reales Marketing mehr Gewicht. Notwendige Veränderungen im Marketing sind grundsätzlich.

\section{Das Change Management für reales Marketing umfasst} folgende Ansätze:

1. Aufmerksamkeit und Engagement des Top-Managements: Der Ansatz des realen Marketing ist abteilungsübergreifend. Es erfolgt eine neue Gewichtung der Marketingaufgaben und der Budgets. Die informationstechnologischen Voraussetzungen müssen zentral entschieden werden. Zudem ist reales Marketing eine grundsätzliche Haltung. wohlwollende Neutralităt genügt nicht. Auch die obersten Manager brauchen den Draht zum Kunden. Das TopManagement ist (einmal mehr) bei dieser Umorientierung. gefordert, diese wichtigen Neuerungen ergeben sich nicht einfach aus dem internen Kräftespiel zwischen Spezialisten. Auch mit der Bekenntnis zur Bottom-up-Vorgehensweise müssen in Unternehmen also manche Voraussetzungen geschaffen werden, die sich nur top-down verwirkli- 
chen lassen. Wichtigste Top-down-Funktion ist dic Leistungsinnovation, die dem Kunden vorausgeht. Das Unternehmen und sein Angebot müssen fur den Kunden substanzicll sein.

2. Neue Fähigkeiten der Verantwortliclıen in Marketing und Vertricb: Ganze Heerscharen von klassischen Marketingbeauftragten und ausgebildeten Marketingfachleuten brauchen neue Ziele, Aufgaben und damit Fähigkeiten im Marketing. Zukünftige Marketingverantwortliche sind eher Ingenieure, Informatiker, Prozess-Spezialisten (zum Beispiel Brinker/McLellan 2014). Sie verlassen den Schutz der schliussigen Markenargumentation. Vertriebsleute suchen den Schulterschluss mit dem Marketing. Gemeinsam führen Marketing und Vertrieb den Kunden zum Kauf, Dazu brauchen wir gleichzeitig mehr Generalisten.

3. Neue Allokation der Budgets: Der Druck auf Budgets der klassischen Markenführung setzt sich fort. Ehenso gilt $\mathrm{es}$, die klassische Marktforschung in der Identifikationswelt des Kunden drastisch zu kürzen. Allerdings genügt es nicht, zu sparen. Fs gilt vielmelır, die Mittel wirksamer im Kundenprozess einzusetzen. Bereits erproben wir eine $\mathrm{Z} u$ teilung der Budgets auf die Hebel im Kundenprozess. Zudem führt der Kampf um Budgets zwischen den Spezialfunktionen des Marketing und Vertriebs zu keinem wirkungsvollen Ergebnis. Die wirksame Budgetallokation stützt sich im Übrigen auch auf die Ergebnisse von cleveren Pilotprojekten, Experimenten und Tests.

4. Neure Erfolgsmessung: Die Messpunkte verlagern sich vom Umsatz auf die relevanten Zwischenschritte im Kaufprozess des Kunden. Nicht einzelne Instrumente werden für sich allein optimiert, sondern Gesamtergebnisse und Ergebnisse der Instrumente und Aktionen werden kombiniert. Nur so lassen sich die Querbeziehungen zwischen Aktivităten (etwa off- und online) angemessen abbilden.

5. Neue Rollen und Organisation (auch international): Organisatorisch gilt es, Marketing und Vertrieb zusammenzuführen - Marketing ist Vertriebsunterstützung, bereitet neue Märkte vor und positioniert das Unternehmen für die Kunden substanziell. Unternehmen bewegen sich so schrittweise zur Kundenorganisation. Key Account Management ist dabei ein Vorreiter, Es gilt, mehr von der Wertschöpfung des Unternehmens auf Kunden zu konzentrieren. Weitere Dimensionen, wie beispielsweise Produkte oder Länder, büßen an Gewicht ein. International lässt sich die Rollenteilung vereinfachen, wenn sich das Head- quarter auf eine maßvolle Markenführung konzentriert und sich die Länder auf kundenprozessorientiertes Marketing fokussieren. Für eine Marketingkonzeption gilt es, sich auf Kunden und ihre Prozesse, Leistungen und Marktbearbeitung (inklusive Kanäle) zu konzentrieren.

Zusammengefasst: Der Weg zum realen Marketing besteht in einem umfassenden Change Management. Unterschiedliche Roadmaps zun realen Marketing als Ziel gilt es zu erproben. Diese Umorientierung ist langfristig, trot $\%$ der verbreiteten Kurslebigkeit in der heutigen Zeit.

\section{Folgerungen}

Klingt ja alles vernünftig. Nur: Was hindert den Zugang zum realen Marketing - weshalb wird es nicht schon lange praktiziert? Dazu zwei Argumente:

- Reales Marketing bedeutete Detailarbeit, in die Tiefe gehen, erforschen, was sich wirklich dem Kauf entgegenstellt und was die Treiber sind - Prozessstellen-genau. Detailarbeit ist jedoch für gestresste Verantwortliche im Marketing vorerst ein bedrohender Vorschlag.

- Die Meinungswelt verdrängt die Fakten. Der Gegensatz von Meinung (die uns alle sofort anspringt, die wir bei jeder $\mathrm{Ge}$ legenheit anderen kundtun) und dem Verhalten, dem tatsächlichen (das nicht Ich-gerecht ist, häufig unreflektiert und aus dem Unbewussten getrieben), ist offensichtlich.

Unser tatsächliches Verhalten rührt meist von Automatismen her, die uns angeboren oder allenfalls an-konditioniert sind; jedenfalls schlummern sie im Unbewussten und drängen einmal angeklickt zu offenem Verhalten. Diese Automatismen setzen sich durch in der reaten Welt - ohne unsere bewusste Meinung zu konsultieren. Diese liefert dann allenfalls noch

\section{Handlungsempfehlungen}

- Erfassen Sie die Schritte der Kunden zum Kauf und definieren Sie kritische Stellen in diesem Prozess.

- Kombinieren Sie kundenprozessorientiertes Marketing und Customer Relationship Management. Folgen. Sie den neuen Regeln des realen Marketing.

- Beherzigen Sie die Grundlagen der Verhaltensforschung und das Handwerk des Direkt- und Internetmarketing, um Kunden zu Handlungen zu bewegen.

- Beschleunigen Sie die Transformation des Marketing. 
die Begriundung nach -, zum Beispicl, wenn ein Marktforscher uns nach dem Warum fragl.

In den Mirketingabteilungen finden wir diese Spaltung wieder. Die Meinungen werden frei geäußert, ausgetauscht und gegenseitig bestätigt: „Auch ich würde so handeln!“ Die Reaktionsmechanismen, die das Verhalten steuern, bleiben urberücksiclıtigt, wenn das Markeling die großen Pläne sclmmiedet und die Werber die kreative Kampagne entwickeln.

Marketing und Vertrieb haben nur eine Aufgabe: den Kunden zum Kauf zu führen. Damit wird der Kundenprozess zum zentraten Gegenstand von Marketing und Vertrieb. Manche aktuclen Diskussionen zu Customer Journey und Experience, Sales Funnel, Customer Storys oder Touch Points scheinen unserem Ansaty ähnlich zu sein. Nur sind diese Ansätze von Marketingverantwortlichen meist nur ausgedacht oder viel zu grob. Wir brauchen Tiefgang, um richtig Prioritäten setzen zu können. Vieldiskutierte Best Practices von Unternehmen beruhen darauf, dass Anbieter die Kundenprozesse neu gestalteten und meistens vereinfachten. Die Palette reicht von Amazon, Kasy.com, Nespresso bis Zara. Finsichtig ist auch, dass industrielle Anbieter sich mit den Kundenprozessen noch weit intensiver betassen müssen, weil diese Prozesse bei il ren Kunden noch komplexer und langer sind.

\section{Literatur}

ls rinker, S./McLellan, L. (2014): '[']e Rise of the Chict Marketing 'lichnologist, in: Harvard business Review, July-August.

Cialdini, R. B. (2010): Die Psychologie des Überæugens, 6. Aufl., Bern

Kahneman, 1). (2012): Schnelles Denken, langsames Denken, Mïnchen.

Marketing Science Institute (2014): 2014-2016 Research Priorities, www nisi.org.

Rutschmann, M. (2005): Kaufprozesse von Konsumenten erketnen und lenken, Mehe Marktanteil mit neuem Marketing, Heidelberg.

SIg * Rutschmann, M. (2013): Abschied vom Branding, Wic man Kunden wirklich ans Kaufen fülort - mit Marketing, das sich an Kaufprozessen orientiert, 2. uiberarb. Aufl., Wiesbaden. (ID): 3549204)

Rutschmann, M./Helz, Ch. (2014); Reales Marketing, Stultgart

Abomnenten des Portals Springer für Professionals erhalten diesen beitrag im Volltext unter www.springerprofessional.de/lD).

Zusätzlicher Verlagsservice für Abonnenten von „Springer für Professionals | Marketing“

Zum Thema "Reales Marketing"

finden Sie unter www.springerprofessional.de 12 Beiträge im Fachgebiet Marketing

\section{Medium}

口 Zeitschriftenartikel (4)

$\square$ Buch (1)

口 Buchkapitel (7)

Sprache

[) Deutsch (12)
Von der Verlagsredaktion empfohlen

Beiz, C., Huber, D, Okonek, C., Rutschmann, M.: Reales Kundenverhalten - reales Marketing, in: Belz, C. (Hrsg.): Innovationen im Kundendialog, Wiesbaden 2011, S.35-67, www.springerprofessional.de/1816248

Binckebanck, L.: Verkauf in schwierigen Zeiten, in: Binckebanck, L.: Verkaufen nach der Krise, Wiesbaden 2011 , \$. 23-42, www.springerprofessional.de/1816482 